\title{
Multi-Continuum Approach to Modeling Shale Gas Extraction
}

\author{
Anna Russian • Philippe Gouze • Marco \\ Dentz • Alain Gringarten
}

\begin{abstract}
Production rates in horizontal shale gas wells display declines that are controlled by the low permeability and the intrinsic heterogeneity of the shale matrix. We present an original multi-continuum approach that yields a physical model able to reproduce the complexity of the decreasing gas rates. The model describes the dynamics of gas rate as function of the physical reservoir parameters and geometry, while the shale matrix heterogeneity is accounted for by a stochastic description of transmissivity field. From the $D=3$ dimensional problem setting, including the heterogeneous shale matrix, the fractures generated by the hydrofracking operations, as well as the production well characteristics, we establish an effective upscaled 1D model for the gas pressures in fracture and matrix as well as the volumetric flux. We analyse the decline curves behaviour and we identify the time scales that characterize the dynamics of the gas rate decline using explicit analytical Laplace space solutions of the upscaled process model. Asymptotically the flux curves decrease exponentially, while in an intermediate regime we find a power law behaviour, in which the flux scales with a power-law in time as $t^{-\beta}$, where $\beta$ reflects the medium heterogeneity. We use this solution to fit a set of real data displaying distinctly different decline trends and study the sensitivity of the model to the reservoir parameters in order to identify their respective controls at the different stages of the decline curve dynamics. Results indicate that the initial value of the gas rate is determined by the transmissivity of the fractures and the initial pressure of the gas in the shale matrix. The latter causes mainly a shift of the entire decline curve. The early time of decline curve shape is primarily controlled
\end{abstract}

Anna Russian

Géosciences, Université de Montpellier 2, CNRS, Montpellier, France.

Tel.: +33 467149315

E-mail: anna.russian@gm.univ-montp2.fr

Philippe Gouze

Géosciences, Université de Montpellier 2, CNRS, Montpellier, France.

Marco Dentz

Institute of Environmental Assessment and Water Research (IDAEA), Spanish National Research Council (CSIC), Barcelona, Spain.

Alain Gringarten

Centre for Petroleum Studies, Imperial College London (ICL), London, United Kingdom. 
by the fracture properties (compressibility and transmissivity). During the main part of the economically valuable production times, i.e. before the production rate drops exponentially, the decline curve is strongly controlled by the properties of the shale-rocks including their heterogeneity, which is modeled by two parameters describing the non-Fickian pressure diffusion effects in a stochastic framework.

Keywords Shale Gas · Modeling · Multi-continuum Model · Shale Reservoirs

\section{Introduction}

Shale gas has become an increasingly important source of natural gas. Shale gas reservoirs contain a large portion of the remaining gas reserves in the world, but are less developed in comparison with conventional gas. The main characteristic of shale gas reservoirs is that they exhibit extremely low matrix permeability. Modelling the dynamic behaviour of shale gas extraction is particularly challenging because the problem includes a variety of physical and chemical processes interrelated at different scales. Moreover, the spatial heterogeneity triggered by fracking operations brings complexity in the gas flow dynamics while the extremely tight formations give rise to long asymptotic decreasing flowrate in the gas production that cannot be explained by classical linear models.

Most of the commonly used methods derive from the pioneer work of Arps, developed for conventional reservoir [1]. Arps rate time model and successive extensions quantify the decline behaviour in terms of the Arps decline exponent $b$. Decline curve analysis is one of the oldest and most used diagnostic tool of petroleum engineer. Arps-derived methods are popular for analyzing decline curves because they require only a 'best fit' with the measured gas rate. However, the use of Arps rate decline equations for unconventional reservoirs often over estimates reserves. The $b$ values obtained by fitting gas production data are greater than 1 , resulting in unrealistic production forecasts with rates that never approach zero. This issue has been widely discussed, e.g. [2,3], and new models have been proposed to avoid these physically unreasonable results, for instance by considering a transient productivity index e.g. [4-6].

Generally, gas production is characterized by successive different regimes: after a first initial regime often difficult to predict because it depends by many different processes (fracturing, flow-back of the water used to fracking, etc), gas enters the effective production regime where the gas flow through a system of microfracture thought the extraction well. During this transient regime the gas rate production curve decreases following different scaling behaviour till it achieves the final regime where the gas production rate decrease exponentially [7]. A limitation of the models mentioned above, is that the development of different flow regimes is difficult to predict which limits their utility for long-term production predictions. Specifically the transient regime preceding the exponential decrease is controlled by coupled effects of both the fractures and the shale matrix properties and can display distinctly different trends.

The aim of this work is to obtain a simplified but effective description of the dynamics of gas production at observation scale, tightly linked to the underlying processes at lower scales. This upscaled model allows extracting the critical information on the reservoir characteristics, which can be used for predicting long-term 
behaviour. Considering the manifest uncertainty on the actual processes and the physical parameters that control the dynamic of gas the extraction, many authors have focused their efforts on developing models as simple as possible.

For instance [8] stated that gas production follows a nearly universal function scaled by only two parameters. The total amount of gas in place and an characteristic fracture interference time, i.e., the time for a pressure pulse to propagate between two planar hydrofracture. The op. cit. model [8] is a non-linear homogeneous diffusion model. It predicts that after few months of production, the recovery rate scales as $t^{-1 / 2}$ until a given characteristic time after which it decreases exponentially. This is the classical behavior observed in dual continuum models for linear solute transport or two-phase flow in highly heterogeneous media [25, 24]. However, there are many examples, as those studied in this paper, of rate curves which do not display the $t^{-1 / 2}$ decline. Yet, shale gas reservoir are naturally heterogeneous and we can anticipate that this heterogeneity must be taken into account for explaining the distinctly different rate curves observed. Moreover we note that the total amount of gas, one of the two parameters of the op. cit. model of [8], is rarely known a priori.

The aim of the present paper is to propose a model that represent the complex, $3 D$ heterogeneous reservoir response with a simplified 1-dimensional linear model, where heterogeneity is handled within a stochastic framework. Specifically, we derive an upscaled multi-continuum model that can explain the long declining rates observed in the analysis of gas shale production

The model is based on the widely used dual porosity approach which is a priori meaningful for such a dual system containing hydraulic discontinuities in less permeable matrix. In dual porosity reservoir models, the shale matrix is the portion of the reservoir that can store large quantities of gas, but displays low conductivity that hamper transporting the gas over long distances. Conversely, the fractures, which partition the matrix, is the main vector for transporting the gas toward the extraction well, for instance. But the fractures have a limited potential for storage.

In conventional reservoir the fractured network can pre-exist (naturally fractured reservoir) but in shale gas reservoirs, the fracture clusters, constituted by fracture, micro-fracture, cracks and natural fractures re-opened, are generated by multiple stages of hydraulic fracturing around horizontal wells [9]. After the fracking stages, the gas diffuses to the production well through the network of fractures, which is recharged by diffusion from the matrix elements. Since the pioneering work of [10], a number of double permeability/porosity models have been developed (e.g. [11-15]) and have been extended to gas production problem even considering heterogeneous matrix (e.g. [16-20]). These models assume that both fractures and matrix elements are in quasi-equilibrium and mass transfer is modelled as a first order process. Linear reservoir models that take into account transient matrix-fracture transfer have been derived in Laplace space by $[12,21]$ and extended by [22].

Here we consider non-equilibrium effects in the matrix triggered by its heterogeneity. Mass exchanges between the fracture system and the heterogeneous matrix give rise to non-integer exponents of the rate-decline slopes and, as an evidence, allow modeling a large range of rate-production data. Transient matrixfracture transfers are controlled by a matrix memory kernel which is defined by the properties of the matrix only. On the other hand we reduce the complexity 
of the problem by considering diffusion in the matrix linear, even though it is, in principle, non-linear. Notice that the validity of linearization of the diffusion problem in the matrix has been favorably discussed in [23-25].

The underlying conceptual model is illustrated schematically in Figure 1. In Section 2, we describe the multi-continuum model, derive the constitutive upscaled effective equations and then give an analytical solution for the pressure and the gas flowrate in the Laplace space. In Section 3 we test this multi-continuum model to actual gas production from a shale gas formation, which displays dissimilar decreasing rate curves. The objective of these examples is to illustrate the ability of the model to explain distinctly different behaviours by the intrinsic properties of the reservoir. Then, we study the sensitivity of the model to the reservoir parameters and identify the set of parameter that primarily control the dynamics of the declining flowrate. Conclusions are given in Section 4.

\section{Multi-Continuum Model}

The model relies on the idea that the gas, initially trapped in the matrix, can be drained by the fractured zone after hydraulic fracturing and diffuse toward the horizontal part of the extraction well. Thus, one can identify two main processes: (i) the refilling of the fractured zone by the matrix, and (ii) the migration of the gas to the extraction points through the fractured zone. Figure 1 illustrates the conceptual model. The model is constituted by a horizontal well at a given depth with a number of vertical fractures $\left(N_{f}\right)$ perpendicular to the well. Each fracture stage is associated to a planar fractured zone, which is characterized by a higher conductivity compared to the surrounding matrix, where the gas is trapped.

The aim is to start from the $d=3$ dimensional radial conceptual model given in Fig. 1 and upscale it into a $d=1$ dimensional equivalent one by using spatial and ensemble averaging. We handle the spatial heterogeneity of the matrix stochastically, with spatially varying conductivities model as spatial random fields. We consider a stationary and ergodic medium with a finite correlation, so that the medium can be completely defined by a single point distribution for an observation scale much larger than the correlation scale.

\subsection{Model Derivation}

\subsubsection{Statement of the problem}

The governing equation for the pressure in the model sketched in figure 1 reads in radial coordinates $[26]$ :

$$
s(z) \frac{\partial p(r, z, t)}{\partial t}-\frac{1}{r} \frac{\partial}{\partial r}\left[r K(r, z) \frac{\partial p(r, z, t)}{\partial r}\right]-\frac{\partial^{2}[K(r, z) p(r, z, t)]}{\partial z^{2}}=0
$$

where $p(r, z, t)$ is the pressure in radial coordinates $(r, z)$ at time $t$, with $r_{w} \leq r \leq$ $R, s(z)$ is the effective compressibility of the system and $K$ is given by $K=k / \mu$ with $k$ the permeability of the system and $\mu$ the viscosity of the gas. The effective 


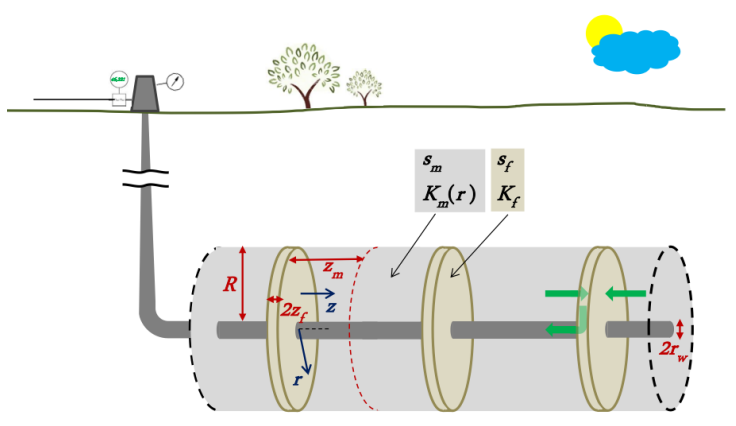

Fig. 1 Sketch of the multi-continuum model. The model is constituted by an horizontal well of radius $r_{w}$ at a depth $h$ with a given number $N_{f}$ of fractures which create fractured zones assumed to be circular, with a radius $R$ and a planar of thickness $z_{f}$. The fractured zone $R$ partition the matrix whose drainable thickness is denoted by $z_{m}$. The radial distance from the axis of the horizontal well is indicated by $r$ and the horizontal distance by $z$. The parameters $s_{f}$ and $s_{m}$ denote the specific storativity of the fracture zone and of the matrix, respectively, $\left[L^{-1}\right]$, while $K_{f}$ and $K_{m}(r)$ denote the respective conductivities [ $\left.L T^{-1}\right]$.

compressibility of the system is given by [26]:

$$
s=(1-\phi) a+\phi b_{i}, \quad a=\frac{1}{1-\phi} \frac{\partial \phi}{\partial p}, \quad b_{i}=\frac{1}{\rho_{i}} \frac{\partial \rho_{i}}{\partial p}
$$

with $a$ and $b_{i}$ the compressibility of the reservoir rock and of the fluid respectively, $\phi$ the porosity and $\rho_{i}$ the density. In case of multi-phase flow the total compressibility is given by: $s=(1-\phi) a+\phi\left(\mathcal{S}_{o} b_{o}+\mathcal{S}_{g} b_{g}+\mathcal{S}_{w} b_{w}\right)$, where $\mathcal{S}$ is the saturation index and the subscripts $o, g, w$, stand for: oil, gas and water [26]. However the multi-phase extension is generally not straightforward because parameters can vary in time.

Equation (1) can be separated into one equation for radial diffusion in the planar fracture and another one for diffusion in the matrix through the fractured zone. For the fracture, we obtain

$$
s_{f} \frac{\partial p_{f}(r, z, t)}{\partial t}-\frac{1}{r} \frac{\partial}{\partial r}\left[r K_{f} \frac{\partial p_{f}(r, z, t)}{\partial r}\right]-K_{f} \frac{\partial^{2} p_{f}(r, z, t)}{\partial z^{2}}=0
$$

where we define $p_{f}(r, z, t)=p(r, z, t)$ for $z \leq z_{f}$ with $z_{f}$ maximum half aperture of the planar fracture, and similarly for $s_{f}$ and $K_{f}$. Here and in the following the subscripts $f$ and $m$ refer to the fractured zone and to the matrix, respectively. We characterize the fractured zone by constant effective fracture storativity $s_{f}$ and conductivity $K_{f}$ arguing that the variability scale of fracture heterogeneity is small such that the fracture homogenizes at times much shorter than the time scales of interest. Symmetry allows us to consider mass exchange between a single fracture matrix system only. The solution for the full system is obtained by multiplication of the solution for the single fracture matrix system by twice the number $N_{f}$ of fracture stages. In the matrix, the pressure behavior is described by

$$
s_{m} \frac{\partial p_{m}(r, z, t)}{\partial t}-\frac{1}{r} \frac{\partial}{\partial r}\left[r K_{m}(r) \frac{\partial}{\partial r} p_{m}(r, z, t)\right]-K_{m}(r) \frac{\partial^{2} p_{m}(r, z, t)}{\partial z^{2}}=0,
$$


where $s_{m}, p_{m}(r)$ and $K_{m}(r)$ indicate respectively effective compressibility, pressure and permeability over viscosity in the matrix for $z_{f} \leq z \leq z_{m}$ and $z_{m}$ the maximum size of matrix influenced by the fracture.

At the interface between the fractured zone and the matrix, at $z=z_{f}$, we impose continuity of pressure and flux:

$$
p_{f}\left(r, z_{f}, t\right)=p_{m}\left(r, z_{f}, t\right),\left.\quad K_{f} \frac{\partial p_{f}(r, z, t)}{\partial z}\right|_{z_{f}}=\left.K_{m}(r) \frac{\partial p_{m}(r, z, t)}{\partial z}\right|_{z_{f}} .
$$

Moreover we consider that the radial flux thought the external edge of the fracture is negligible because $R$ indicates the radius of influence of the horizontal well which determines the Stimulated Reservoir Volume (SRV). Gas trapped at a distance $r>R$ from the horizontal well is not mobilized during production [8]. Thus, we set a no-flux condition at the edge of the fracture, $K_{f}\left[\partial p_{f}(r, z, t) / \partial r\right]_{r=R}=$ 0 . The same boundary condition is imposed in the matrix domain at $z=z_{m}$, $K_{m}(r)\left[\partial p_{m}(r, z, t) / \partial z\right]_{z=z_{m}}=0$.

The permeability in the fracture is much larger than the permeability in the matrix, thus $K_{f} \gg \max \left\{K_{m}(r)\right\}$. Therefore, equilibrium along the $z$ direction in the fracture is reached relatively faster than in the matrix and we can suitably assume $p_{f}(r, z, t) \simeq p_{f}(r, t)$. Moreover, the difference in conductivity between fracture and matrix makes matrix diffusion preferably oriented in the direction of the fractures. Thus, neglecting radial diffusion in the matrix, the constitutive equation for the matrix given in Eq. (4) reduces to:

$$
s_{m} \frac{\partial p_{m}(r, z, t)}{\partial t}-K_{m}(r) \frac{\partial^{2} p_{m}(r, z, t)}{\partial z^{2}}=0 .
$$

We assume that the matrix conductivity varies in radial direction. The matrix acts as a source term for the fractures as expressed by the continuity conditions (5).

\subsubsection{Spatial average}

In order to obtain a simpler effective description, we integrate the governing diffusion equation over the the coordinate $z$, and we reduce the $d=3$ dimensional radial problem into an effective $d=1$ dimensional one. The average pressures in the fracture and in the matrix are defined as

$$
\bar{p}_{f}(r, t)=\frac{1}{z_{f}} \int_{z_{0}}^{z_{f}} d z p_{f}(r, z, t), \quad \bar{p}_{m}(r, t)=\frac{1}{z_{m}} \int_{z_{f}}^{z_{m}+z_{f}} d z p_{m}(r, z, t) .
$$

By executing spatial average of Eq. (3), as defined in 7, over the fracture aperture $z_{f}$, gives:

$$
S_{f} \frac{\partial \bar{p}_{f}(r, t)}{\partial t}-\frac{1}{r} \frac{\partial}{\partial r} r T_{f} \frac{\partial \bar{p}_{f}(r, t)}{\partial r}=\left.K_{m}(r) \frac{\partial p_{m}(r, z, t)}{\partial z}\right|_{z=z_{f}}
$$

where we used the continuity condition (5) and we defined the total compressibility of the fractured zone $S_{f}=s_{f} z_{f}$ and the effective transmissivity $T_{f}=K_{f} z_{f}$. The last term in Eq. (8) is the flux at the interface between fracture and matrix and it is obtained from the solution of the diffusion equation in the matrix, which is 
given in Eq.(6). We solve Eq.(6) in the Laplace space with the following boundary conditions: continuity of pressure $p_{m}(r, z, t)=\bar{p}_{f}(r, t)$ at $z=z_{f}$ and no flux condition boundary condition at $z=z_{m}$. Notice that we set the fracture pressure at the interface equal to the cross-sectional average, which is justified because the vertical homogenization time over the fracture width $t_{f}=z_{f}^{2} s_{f} / K_{f}$ is much smaller than the characteristic matrix time scales.

Using the method of Green functions, we obtain the average pressure in the matrix as a function of the pressure in the fractured zone:

$$
\begin{aligned}
\bar{p}_{m}(r, t) & =\int_{0}^{t} d \tau g\left[t-\tau, \tau_{m}(r)\right] \bar{p}_{f}(r, \tau) \\
& -\bar{p}_{m}(r, 0) \int_{0}^{t} d \tau g\left[\tau, \tau_{m}(r)\right]+\bar{p}_{m}(r, 0),
\end{aligned}
$$

with the uniform in $z$ initial condition $\bar{p}_{m}(r, t=0)=p_{m}(r, t=0)$. The characteristic time scale for pressure propagation within the matrix is

$$
\tau_{m}(r)=z_{m}^{2} s_{m} / K_{m}(r)
$$

In Eq. $(9), \bar{p}_{m}(r, 0)$ is the initial pressure in the matrix and the matrix memory kernel $g\left(t, \tau_{m}\right)$ is obtained by averaging the solution of Eq. (6) for the pulse boundary condition $p_{m}(r, z, t)=\delta(t)$ at the interface $z=z_{f}$. The function $g\left(t, \tau_{m}\right)$ is computed, for simplicity, in Laplace space. Laplace transform of $g\left(t, \tau_{m}\right)$, with respect to the time $t$, reads as $[27,28]$ :

$$
g^{*}\left[\lambda, \tau_{m}(r)\right]=\frac{1}{\sqrt{\lambda \tau_{m}(r)}} \tanh \left[\sqrt{\lambda \tau_{m}(r)}\right]
$$

where $\lambda$ is the Laplace variable and the asterisk denotes function in Laplace space. Laplace transform is defined in [29]. For times $t \ll \tau_{m}(r)$ the memory function $g\left(t, \tau_{m}\right)$ decreases with time as $g \propto t^{-1 / 2}$, while it is cut-off exponentially for $t>\tau_{m}$.

In order to obtain the flux term appearing on the right side of Eq. (8) as a function of $\bar{p}_{f}$, we integrate along the $z$ direction Eq.(6), we transform it in the Laplace space and we substitute $\bar{p}_{m}$ as a function of $\bar{p}_{f}$ according to Eq. (9). Thus, the spatial derivative on the right side of Eq. (8) reads in Laplace space as:

$$
\left.\frac{\partial p_{m}^{*}(r, z, \lambda)}{\partial z}\right|_{z=z_{f}}=-\frac{\lambda z_{m} s_{m}}{K_{m}} g^{*}\left[\lambda, \tau_{m}(r)\right]\left[\bar{p}_{f}^{*}(r, \lambda)-\frac{\bar{p}_{m}(r, 0)}{\lambda}\right] .
$$

Using this result in Eq.(8), we obtain the following non-local closed form equation for the pressure in the fracture:

$$
\begin{aligned}
\lambda\left\{S_{f}+S_{m} g^{*}\left[\lambda, \tau_{m}(r)\right]\right\} \bar{p}_{f}^{*}(r, \lambda)-\frac{T_{f}}{r} \frac{\partial}{\partial r} r \frac{\partial \bar{p}_{f}^{*}(r, \lambda)}{\partial r}= \\
S_{f} \bar{p}_{f}(r, 0)+S_{m} g^{*}\left[\lambda, \tau_{m}(r)\right] \bar{p}_{m}(r, 0)
\end{aligned}
$$

where $S_{m}=s_{m} z_{m}$. Considering that at time zero $t=0$ the fractured zone is in equilibrium with the matrix $\bar{p}_{f}(r, 0)=\bar{p}_{m}(r, 0)$ the previous equation reduces to:

$$
\begin{aligned}
\lambda\left\{S_{f}+S_{m} g^{*}\left[\lambda, \tau_{m}(r)\right]\right\} \bar{p}_{f}^{*}(r, \lambda)-\frac{T_{f}}{r} \frac{\partial}{\partial r} r \frac{\partial \bar{p}_{f}^{*}(r, \lambda)}{\partial r}= \\
\left\{S_{f}+g^{*}\left[\lambda, \tau_{m}(r)\right]\right\} \bar{p}_{f}(r, 0) .
\end{aligned}
$$


Note that if one considers a homogeneous matrix, so that the model reduces to a double porosity medium, the memory function is time dependent only, i.e. $\bar{g}(t \mid r) \equiv \bar{g}(t)$. The function $g(t)$, obtained by solving Eq.(6) in Laplace space, represents the boundary matrix flux. One note that $g(t)$ is equivalent to the Laplace transform of the gas flux in the linearized model of [8].

In the following, we consider an heterogeneous matrix and thus we average the flux over the fracture-matrix boundary $r$. We use a stochastic approach because we do not have a proper description of the heterogeneity distribution along $r$. Accordingly, we describe the heterogeneity of the matrix by a distribution of transition times, as described in the next section.

\subsubsection{Ensemble average}

In order to obtain an upscaled effective formulation for the multi-continuum model, we average the governing equation (13) over the heterogeneity of the matrix and we obtain:

$$
\lambda\left[S_{f}+\varphi^{*}(\lambda)\right]\left\langle\bar{p}_{f}^{*}(r, \lambda)\right\rangle-\frac{T_{f}}{r} \frac{\partial}{\partial r}\left[r \frac{\partial\left\langle\bar{p}_{f}^{*}(r, \lambda)\right\rangle}{\partial r}\right]=\left[S_{f}+\varphi^{*}(\lambda)\right]\left\langle\bar{p}_{f}(r, 0)\right\rangle,
$$

where the squared brackets indicate the ensemble averaged quantities. In Eq. (15) we defined the global memory function as:

$$
\varphi^{*}(\lambda)=\left\langle S_{m} \bar{g}^{*}\left[\lambda, \tau_{m}(r)\right]\right\rangle
$$

where the angular brackets indicate ensemble average over the distribution of characteristic time scales $\tau_{m}(r)$. The global memory function for an heterogeneous medium is given by the superposition of single memory functions $g(t)$ for homogeneous matrix.

In order to average Eq. (13) and obtain Eq. (15) we use the mean field approximation:

$$
\left\langle\varphi^{*}(\lambda) \bar{p}_{f}^{*}(r, \lambda)\right\rangle \simeq\left\langle\varphi^{*}(\lambda)\right\rangle\left\langle\bar{p}_{f}^{*}(r, \lambda)\right\rangle
$$

which is pertinent for times at which the variation scale of the pressure in the fracture, $\bar{p}_{f}$, is larger than the heterogeneity scale of matrix. Note that this approximation is done on a routine basis in similar multi-rate mass transfer (MRMT) models for solute transport in multi-continuum models $[27,28]$. The global memory function defined in Eq. (16) is given by superposition of the local memory functions $\mathrm{n} g(\tau, t)$ :

$$
\varphi(t)=S_{m} \int_{0}^{\infty} P(\tau) g(t, \tau) d \tau
$$

where $P(\tau)$ is the probability density function of characteristic times $\tau_{m}$, which are related to the immobile conductivity $K$, through Eq. (10). The distribution of characteristic time $\tau_{m}$ is expressed in terms of the distribution of $K_{m}, P_{K}(k)$, as: $P(\tau)=z_{m}^{2} s_{m} / \tau^{2} P_{K}\left(z_{m}^{2} s_{m} / \tau\right)$. A broad distribution of mass transfer scales may 
be modeled by a truncated power law distribution of the trapping times in the matrix:

$$
P(\tau)=\frac{1-\beta}{\tau_{2}^{1-\beta}-\tau_{1}^{1-\beta}} \tau^{-\beta} \Theta\left(\tau_{2}-\tau\right) \Theta\left(\tau-\tau_{1}\right),
$$

where $1 / 2<\beta<1 ; \tau_{1}$ and $\tau_{2}$ are the lower and upper limits of the truncated power law distribution, and $\Theta(\cdot)$ is the Heaviside step function, which is equal to one if its argument is larger than zero and zero otherwise. We insert Eq. (19) in Eq. (18) and, as shown in the Appendix A, we approximate the global memory function by the following truncated power-law:

$$
\varphi(t)=\frac{S_{m}}{\tau_{2} \Gamma(1-\beta)}\left(\frac{t}{\tau_{2}}\right)^{-\beta} \exp \left(-\frac{t}{\tau_{2}}\right),
$$

where $\Gamma(\cdot)$ denotes the gamma function. In Laplace space the memory function reads as:

$$
\varphi^{*}(\lambda)=S_{m}\left(\lambda \tau_{2}+1\right)^{\beta-1}
$$

From Eq.(15), we note that the power law distribution of $\tau$ controls the dynamic of the system when $\varphi^{*}(\lambda)$ is larger that $S_{f}$. This condition identifies a characteristic time $\tau_{a}$ given by

$$
\tau_{a}=\tau_{2}\left(\frac{S_{f}}{S_{m}}\right)^{\frac{1}{1-\beta}}
$$

which corresponds to the activation time of the matrix. Specifically, it denotes the time for which $S_{m}\left(\tau_{a} / \tau_{2}\right)^{1-\beta}=S_{f}$. The influence of the truncated power law distribution of trapping time in the matrix is dominant for time $t>\tau_{a}$. Note that for $t<\tau_{a}$ the model gives information on the fractured zone only and not on the reservoir properties. Only for time $t>\tau_{a}$ the flux derived in the proposed model depends on the properties of the reservoir and then we can obtain different behaviour according to parameters of the matrix.

\subsection{Solutions}

In the following we solve Eq. (15) in Laplace space. The general solution of Eq. (15) is given by (see Appendix B for details):

$$
\left\langle\bar{p}_{f}^{*}(r, \lambda)\right\rangle=\left[\mathcal{I}_{1}\left(R^{\prime}\right) \mathcal{K}_{0}\left(r^{\prime}\right)+\mathcal{K}_{1}\left(R^{\prime}\right) \mathcal{I}_{0}\left(r^{\prime}\right)\right] C^{*}(\lambda)+\frac{\bar{p}_{f}(0)}{\lambda}
$$

where $\mathcal{I}_{0}(\cdot)$ and $\mathcal{K}_{0}(\cdot)$ are the 0 -th order modified Bessel functions of first and second kind respectively, $\mathcal{I}_{1}(\cdot)$ and $\mathcal{K}_{1}(\cdot)$ are the 1-st order modified Bessel functions of first and second kind; $C^{*}(\lambda)$ is determined by the boundary conditions. We furthermore defined:

$$
R^{\prime}(\lambda)=R \sqrt{\tau^{\prime}(\lambda) \lambda}, \quad \quad r^{\prime}(\lambda)=r \sqrt{\tau^{\prime}(\lambda) \lambda}
$$




$$
\tau^{\prime}(\lambda)=\frac{S_{f}+\varphi^{*}(\lambda)}{T_{f}}
$$

In order to solve Eq. (15) and compute the $C^{*}(\lambda)$ in Eq. (23), we note that the outgoing flux, at $r=r_{w}$, is proportional to the difference of pressure between the fractured zone and the well. Thus, we impose a Cauchy-type boundary condition at the horizontal well

$$
\begin{aligned}
2 \pi r_{w} T_{f}\left[\frac{\partial\left\langle\bar{p}_{f}(r, t)\right\rangle}{\partial r}\right]_{r=r_{w}}= & \frac{1}{\alpha}\left[\left\langle\bar{p}_{f}\left(r_{w}, t\right)\right\rangle-p_{w}(t)\right], & r=r_{w} \\
& {\left[\frac{\partial\left\langle\bar{p}_{f}(r, t)\right\rangle}{\partial r}\right]_{r=R}=0, } & r=R
\end{aligned}
$$

where $\alpha$ is the skin factor that relates the pressure drop with the flux of gas entering the horizontal well, and $p_{w}(t)$ is the pressure in the horizontal well. At the edge of the fractured zone, for $r=R$, we imposed a no-flux boundary condition as discussed in Sect. 2.1.1. Thus, the function $C^{*}(\lambda)$ in Eq.(23) is given by:

$C^{*}(\lambda)=-\frac{\frac{\bar{p}_{f}(0)}{\lambda}-p_{w}^{*}(\lambda)}{\mathcal{I}_{0}\left(r_{w}^{\prime}\right) \mathcal{K}_{1}\left(R^{\prime}\right)+\mathcal{I}_{1}\left(R^{\prime}\right) \mathcal{K}_{0}\left(r_{w}^{\prime}\right)+A r_{w}^{\prime}\left[\mathcal{I}_{1}\left(R^{\prime}\right) \mathcal{K}_{1}\left(r_{w}^{\prime}\right)-\mathcal{I}_{1}\left(r_{w}^{\prime}\right) \mathcal{K}_{1}\left(R^{\prime}\right)\right]}$,

where we define $A=2 \pi T_{f} \alpha$ and, similarly as in Eq. (24),

$$
r_{w}^{\prime}=r_{w} \sqrt{\tau^{\prime}(\lambda) \lambda}
$$

The flux of gas entering the horizontal well, $Q(t),\left[L^{3} T^{-1}\right]$, is computed as the radial derivative of the pressure at $r=r_{w}$ and is given by:

$$
Q(t)=-2 \pi r_{w} T_{f}\left[\frac{\partial\left\langle\bar{p}_{f}(r, t)\right\rangle}{\partial r}\right]_{r=r_{w}}
$$

Hence, taking the radial derivative of Eq. (23), one obtains:

$$
Q^{*}(\lambda)=2 \pi r_{w}^{\prime} T_{f}\left[\mathcal{I}_{1}\left(R^{\prime}\right) \mathcal{K}_{1}\left(r_{w}^{\prime}\right)-\mathcal{K}_{1}\left(R^{\prime}\right) \mathcal{I}_{1}\left(r_{w}^{\prime}\right)\right] C^{*}(\lambda) .
$$

with $C^{*}(\lambda)$ given in Eq. (28). Substituting expression $(28)$ for $C^{*}(\lambda)$ in the previous equation yields:

$Q^{*}(\lambda)=\frac{2 \pi r_{w}^{\prime} T_{f}\left[\frac{\bar{p}_{f}(r, 0)}{\lambda}-p_{w}^{*}(\lambda)\right]\left[\mathcal{I}_{1}\left(R^{\prime}\right) \mathcal{K}_{1}\left(r_{w}^{\prime}\right)-\mathcal{K}_{1}\left(R^{\prime}\right) \mathcal{I}_{1}\left(r_{w}^{\prime}\right)\right]}{\mathcal{I}_{0}\left(r_{w}^{\prime}\right) \mathcal{K}_{1}\left(R^{\prime}\right)+\mathcal{I}_{1}\left(R^{\prime}\right) \mathcal{K}_{0}\left(r_{w}^{\prime}\right)+A r_{w}^{\prime}\left[\mathcal{I}_{1}\left(R^{\prime}\right) \mathcal{K}_{1}\left(r_{w}^{\prime}\right)-\mathcal{I}_{1}\left(r_{w}^{\prime}\right) \mathcal{K}_{1}\left(R^{\prime}\right)\right]}$ 
2.3 Asymptotic behaviour

In order to depict the long time behaviour of the model we perform an asymptotic expansion of (32) for the limit $\lambda \tau^{\prime} \ll 1$. Thus, we obtain

$$
Q^{*}(\lambda) \approx \frac{\tau^{\prime}(\lambda) \pi T_{f}\left(R^{2}-r_{w}^{2}\right) \Delta p}{1+\lambda \tau^{\prime}(\lambda)\left[\frac{R^{2}}{2} \ln \frac{R}{r_{w}}+\frac{A}{2}\left(R^{2}-r_{w}^{2}\right)\right]}
$$

as outlined in detail in Appendix C. Note that for simplicity we set the pressure at the well $p_{w}^{*}(\lambda)$ constant and $\Delta p=p_{0}-p_{w}$.

It is worth noticing that for times $t \gg \tau_{2}$, or equivalently for $\lambda \tau_{2} \ll 1$, the memory function $\varphi^{*}(\lambda) \rightarrow S_{m}$. Thus, inserting this result in Eq. (25) we observed that $\tau^{\prime}(\lambda)$ tends towards the constant value

$$
\tau^{\prime}(\lambda) \rightarrow \frac{S_{f}+S_{m}}{T_{f}} .
$$

Moreover $\tau^{\prime}(\lambda)$ is also constant when $\varphi^{*}(\lambda) \ll S_{f}$ or equivalently for $t \ll \tau_{a}$ (see Eq. $(22))$. In this case, the flux comes from the fractured zone only, $\tau^{\prime}(\lambda)$ tends towards the constant value

$$
\tau^{\prime}(\lambda) \rightarrow \frac{S_{f}}{T_{f}}
$$

and consequently the dynamic of the flowrate depends on the parameters of the fractured zone only. Accordingly, for the two time regimes defined by $t \gg \tau_{2}$ and $t \ll \tau_{a}$ the model behaves as an equivalent homogeneous model with $\tau^{\prime}(\lambda)$ constant and given by (34) and (35), respectively. For these regimes, it follows from the inverse Laplace transform of (33) that the flux behaves exponentially as

$$
Q(t) \sim \exp \left(-t / \tau_{c}\right)
$$

where the time scale $\tau_{c}$ is given by

$$
\tau_{c}=\frac{R^{2} S_{f}}{T_{f}}\left[\frac{1}{2} \ln \frac{R}{r_{w}}+\frac{A}{2}\left(1-\frac{r_{w}^{2}}{R^{2}}\right)\right]
$$

for $t \ll \tau_{a}$ and by

$$
\tau_{c}=\frac{R^{2}\left(S_{f}+S_{m}\right)}{T_{f}}\left[\frac{1}{2} \ln \frac{R}{r_{w}}+\frac{A}{2}\left(1-\frac{r_{w}^{2}}{R^{2}}\right)\right]
$$

for $t \gg \tau_{2}$.

In the intermediate regime $\tau_{a} \ll t \ll \tau_{2}$, the function $\tau^{\prime}(\lambda)$ behaves as

$$
\tau^{\prime} \approx \frac{S_{m}}{T_{f}}\left(\lambda \tau_{2}\right)^{\beta-1} .
$$

By inserting this result in Eq.(33) one find that the Laplace transform of the flux scales as $Q^{*}(\lambda) \propto\left(\lambda \tau_{2}\right)^{\beta-1}$. This implies that the flux in the intermediate time regime decreases with the power-law behaviour

$$
Q(t) \propto\left(\frac{t}{\tau_{2}}\right)^{-\beta}
$$


a)

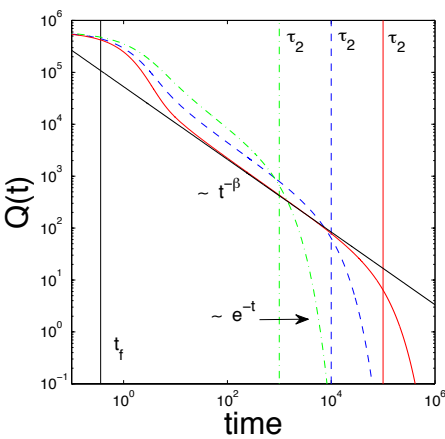

b)

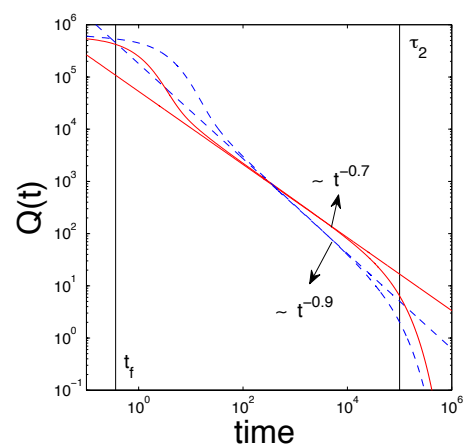

Fig. 2 Temporal evolution of flux as defined in Eq.32 for different values of the cutoff time $\tau_{2}$ (Fig.2a) and different values of $\beta$ (Fig.2b). Parameters: $r_{w}=0.1 \mathrm{~m}, N_{f}=44, R=25 \mathrm{~m}$, $S_{f}=10^{-7} \mathrm{~m} / \mathrm{Pa}, T_{f}=2 \cdot 10^{-9} \mathrm{~m}^{3} / \mathrm{Pa} / \mathrm{s}, \Delta p=3 \cdot 10^{7} \mathrm{~Pa}, S_{m}=10^{-6} \mathrm{~m} / \mathrm{Pa}, \alpha=1 \mathrm{Kg} / \mathrm{m}^{3} / \mathrm{s}$. For Fig. $2 \mathrm{a}: \beta=0.7, \tau_{2}=10^{5} \mathrm{~d}$ (red continuous line), $\tau_{2}=10^{4} \mathrm{~d}$ (blue dashed line) and $\tau_{2}=10^{3} \mathrm{~d}$ (green dash dotted line). For Fig. $2 \mathrm{~b}: \tau_{2}=10^{5} \mathrm{~d}, \beta=0.7$ (red continuous line), $\beta=0.9$ (blue dashed line).

For $\beta=0.5$, which is characteristic for mass transfer between the fracture and a homogeneous matrix domain, the flux scales as $Q(t) \propto\left(t / \tau_{2}\right)^{-1 / 2}$. As outlined in the Introduction, this is the classical flux behavior observed in dual-continuum flow and transport models such as the one employed by [8]. The model proposed here accounts for a heterogeneous matrix domain, which may give rise to different scaling behaviours as predicted by (40).

Figure $2 \mathrm{a}$ and Figure $2 \mathrm{~b}$ illustrate the temporal evolution of the flux $Q(t)$ for different $\tau_{2}$ and values of the exponent $\beta$ in (19). The curves are obtained by numerical inverse Laplace transform of (32) using the commercial software package MATLAB. The gas flux starts decreasing for times larger than $t_{f}=R^{2} S_{f} / T_{f}$, which is the characteristic time for pressure propagation across the fractured zone, or equivalently, the mean depletion time of the fractured zone. In the absence of a matrix region, i.e., for a homogeneous fractured zone only, $t_{f}$ marks the characteristic depletion time scale for exponential decay of the gas flux. In the intermediate time regime $\tau_{a} \ll t \ll \tau_{2}$ the gas flux behaves as a power-law as predicted by (40). We observe an exponential decline of the gas flux for $t \gg \tau_{2}$, as predicted by (36).

\section{Application}

In the following we use the derived multi-continuum model for the interpretation of actual shale gas production data. The model was implemented using the commercial software package MATLAB (MATLAB R2013a, The MathWorks Inc., Natick, MA, 2013). The evolution of the gas flux is determined by numerical inverse Laplace transform of the explicit Laplace space expression (33).

The implemented model computes the inverse Laplace transform. On the basis of this solution, the model parameters are estimated by minimizing the squared relative error between field data and the numerically inverted model. For the parameter estimation, we adapted a MATLAB code that is part of the CTRW 
optimization Toolbox $[31,32]$. The code is based on the routine fminsearch implemented in MATLAB, which uses a Nelder-Mead simplex direct search algorithm to minimize the non-linear least squares objective function. The model allows estimating the following parameters: the radius of the horizontal well $r_{w}$, the mean radius of the zone of influence of the fracture stage $R$, the total compressibility of the fractured zone $S_{f}=z_{f} s_{f}$, the mean effective transmissivity of the fractured zone $T_{f}$, the initial pressure of the reservoir $\bar{p}(0)$, the total compressibility of the matrix $S_{m}=z_{m} s_{m}$, the exponent of the power law distribution of transition times in the matrix given in (19) $\beta$ and its cut off $\tau_{2}$, the number of active fractured zones $N_{f}$, the skin factor $\alpha$. Because of the large number of fitting parameters, the inversion can be not unique. For this reason uncertainties are largely decreased if a prior geological and/or physical information of the site are available in order to fix some parameters and, or, impose a pertinent range of variability on the others. A better knowledge of fracture properties from geophysical data will improve the best fitting of the short time behavior, while (multi-scale) measurements of the matrix dispersivity will improve long term predictions. However these parameters are rarely analyzed and we believe that it is a major issue for reliable predictions.

\subsection{Results}

In this section we consider the gas flowrate of three production wells in Louisiana (US) for illustrating the use of the model. These wells display distinctly different decline curve shapes. As for many other data set, only few information about the medium properties are accompanying the decline curves. Possible modifications of the well head configuration (e.g. change of the setup used to regulate the well head pressure) were not reported as well. Parameters for gas production of the three production wells (noted hereafter A, B and C) are estimated, using as much as possible the information available at this site. The gas flowrate is fitted (see Section 3) by fixing the radius of the horizontal well $r_{w}$ and the number of fractured stages $N_{f}$ expected during the fracking stage. The depth at which the horizontal well was drilled is known and can be used to evaluate the initial pressure $\left(p_{0}\right)$.

Due the large uncertainties on the well pressure value, we considered a constant flowing pressure in the well as the boundary condition $p_{w}(t)=p_{w}$ in $(26)$, and we fit the difference of the initial reservoir pressure and the pressure in the well $\Delta p=\bar{p}(0)-p_{w}$. The results are shown in Figure 3 for well A and Figures $4 \mathrm{a}$ and $4 \mathrm{~b}$ for well $\mathrm{B}$ and $\mathrm{C}$ respectively.

Figure 3 displays the fit for well A gas flowrate with the multi-continuum model (red solid line) and the curves related to equivalent homogeneous models. Similar plots are given for well B and C in figures 4 . The proposed figures are in log-log scale in order to highlight the asymptotic decreasing flowrate. An equivalent homogeneous model that represents the hydraulic properties of the fractured zone only is obtained by setting $\varphi^{*}(\lambda)=0$ in (15). An equivalent homogeneous model that comprises both the fractured and matrix domain is obtained by setting $\varphi^{*}(\lambda)=S_{m}$ in (15). Note that in the asymptotic long time limit $t \gg \tau_{2}$, the memory function reduces to $\lim _{t \rightarrow \infty} \varphi(t)=S_{m} \delta(t)$. The first case represents the behaviour of the homogeneous fracture domain only, while the second case represents the the behaviour of an equivalent homogeneous system when fractures zone and matrix are in equilibrium. For the equivalent homogeneous cases, one can 


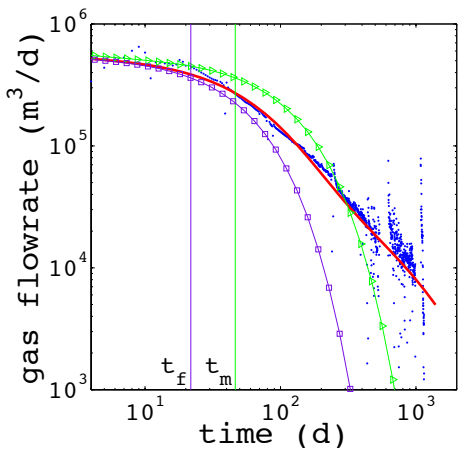

Fig. 3 Gas rate production of well A (blue dots) in log-log scale. Measurements are taken daily. Red line: Multi-continuum model fit. Violet line with squares: equivalent homogeneous model with $\varphi=0$, Green line with triangles: equivalent homogeneous model with $\varphi=S_{m}$. Fixed parameters: $r_{w}=0.09 \mathrm{~m}, N_{f}=44$. Fitted parameters: $R=21.9 \mathrm{~m}, S_{f}=3.2 \cdot 10^{-6} \mathrm{~m} / \mathrm{Pa}$, $T_{f}=8.18 \cdot 10^{-10} \mathrm{~m}^{3} / \mathrm{Pa} / \mathrm{s}, \Delta p=6.6 \cdot 10^{7} \mathrm{~Pa}, S_{m}=3.62 \cdot 10^{-6} \mathrm{~m} / \mathrm{Pa}, b=0.60, \tau_{2}=1.50 \cdot 10^{3} \mathrm{~d}$, $\alpha=2.6 \mathrm{Kg} / \mathrm{m}^{4} / \mathrm{s}$. Timescales: $t_{f}=R^{2} S_{f} / T_{f}=21.8 d$ and $t_{m}=R^{2}\left(S_{f}+S_{m}\right) / T_{f}=46.35 d$.

observe that the gas flowrate deceases exponentially, see Appendix C. The data, however, displays a much slower decrease.

The multi-continuum model can properly fit the experimental data while this is not possible with the equivalent homogeneous models whatever its parametrization. The curves corresponding to the homogeneous models coincide with the multi-continuum model at short times but then decrease faster than what would be required to reproduce the flowrate tailing. The curve representing the fracture domain only decreases exponentially fast for times larger than $t_{f}=R^{2} S_{f} / T_{f}$ which, as pointed out above, is the mean depletion time of the fractured zone. The curve representing the homogeneous equivalent to fractured and matrix domain decreases exponentially fast for times larger than $t_{m}=R^{2}\left(S_{f}+S_{m}\right) / T_{f}$. This time scale represents the characteristic time to deplete both the fracture and matrix zones. These time scales are also reflected in characteristic scales (37) and (38). The multi-continuum model accounts for pre-asymptotic non-equilibrium between matrix and fractured zones and yields a slowly decreasing asymptotic flowrate.

The parameters obtained by the fitting procedure are summarized in Table 1 together with the root mean squared errors of the fits and the correlation coefficients which quantify the good fit between the data and the model. The values of the skin factor $\alpha$ are not mentioned in the table because the data fits are unchanged by varying $\alpha$ in the range of values considered (see later discussion in Section 3.2). Similarly, the value of $\tau_{1}$ is not mentioned because it can be neglected as explained in detail in Appendix A.

The values of the physical parameters obtained by the fitting procedure are in the range of the typical values found in the literature for a shale reservoirs [33-35, 8]. In the fitting process we impose a range of validity for some parameters such as, for example, the radius of the fractured zone $R$ that cannot be larger than the half thickness of the reservoir and some appropriate limits on compressibility, permeability and viscosity. 


\begin{tabular}{llll}
\hline Data & $\mathrm{A}$ & $\mathrm{B}$ & $\mathrm{C}$ \\
\hline $\mathrm{R},[\mathrm{m}]$ & 21.9 & 33.7 & 34.6 \\
$S_{f}=z_{f} s_{f},[\mathrm{~m} / \mathrm{Pa}]$ & $3.2 \cdot 10^{-6}$ & $5.3 \cdot 10^{-6}$ & $3.6 \cdot 10^{-6}$ \\
$T_{f}=z_{f} \frac{k}{\mu},\left[\mathrm{m}^{3} / \mathrm{Pa} / \mathrm{s}\right]$ & $8.2 \cdot 10^{-10}$ & $6.4 \cdot 10^{-9}$ & $1.4 \cdot 10^{-9}$ \\
$\Delta p=\bar{p}_{0}-p_{w},[\mathrm{~Pa}]$ & $6.6 \cdot 10^{7}$ & $8.2 \cdot 10^{6}$ & $3.6 \cdot 10^{7}$ \\
$S_{m}=z_{m} s_{m},[\mathrm{~m} / \mathrm{Pa}]$ & $3.6 \cdot 10^{-6}$ & $2.1 \cdot 10^{-5}$ & $3.1 \cdot 10^{-5}$ \\
$\beta$ & 0.6 & 0.67 & 0.5 \\
$\tau_{2},[$ day $]$ & $1.5 \cdot 10^{3}$ & $6.4 \cdot 10^{2}$ & $4.9 \cdot 10^{4}$ \\
$t_{f},[$ day] & 21.8 & 10.8 & 35.6 \\
$t_{m},[$ day $]$ & 46.4 & 54.1 & 342 \\
RMSE & $6.7 \cdot 10^{3}$ & $6.6 \cdot 10^{3}$ & $1.0 \cdot 10^{4}$ \\
Corr & 0.972 & 0.970 & 9.60 \\
\hline
\end{tabular}

Table 1 Result fitted parameters. Parameters: $R$ radius of the fractured zone, effective compressibility $S_{f}=z_{f} s_{f}$ and $S_{m}=z_{m} s_{m}$ with $z_{f}, z_{m}$ thickness of the fractured zone and the matrix, $s_{f}, s_{m}$ compatibilities given by the soil and the gas compressibility (see Eq.(2)), $T_{f}=z_{f} k / \mu$ with $k$ permeability and $\mu$ viscosity at reservoir conditions, difference of initial pressure and pressure in the well $\Delta p=\bar{p}_{0}-p_{w}$, exponent $\beta$ and cut off $\tau_{2}$ of the power law distribution of Eq.(21); RMSE is the root mean squared error and Corr is the correlation coefficient.
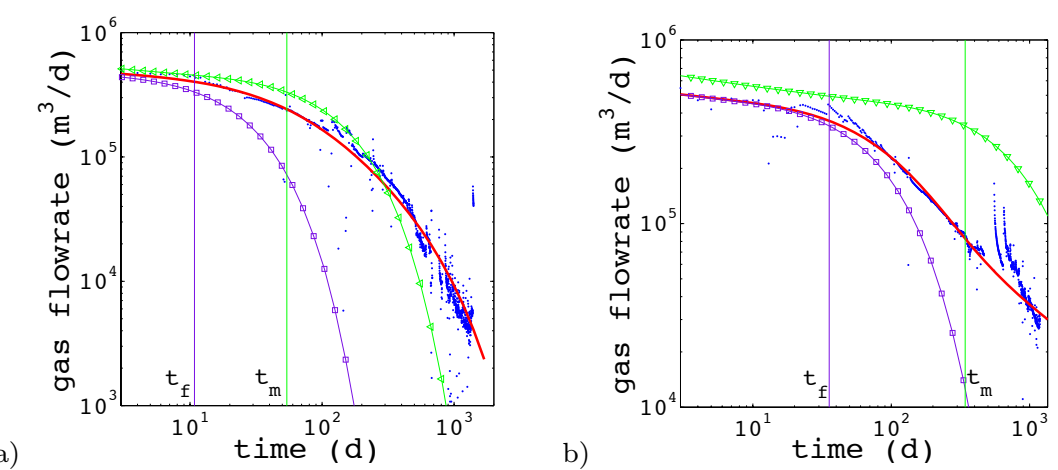

Fig. 4 Gas Rate production of well B (on the left, Fig. 4a) and of well C (on the right, Fig. 4b). Field measurement (blue dots) are taken daily. Violet line with squares: equivalent homogeneous model with $\varphi=0$, green line with triangles: equivalent homogeneous model with $\varphi=S_{m}$. Model parameters for well B (red line): fixed parameters: $r_{w}=0.09 \mathrm{~m}, N_{f}=45$; fitted parameters: $R=33.7 \mathrm{~m}, S_{f}=5.3 \cdot 10^{-6} \mathrm{~m} \mathrm{~Pa}^{-} 1, T_{f}=6.4 \cdot 10^{-9} \mathrm{~m}^{3} \mathrm{~Pa}^{-1} \mathrm{~s}^{-1}, \Delta p=8.2 \cdot 10^{6} \mathrm{~Pa}$, $S_{m}=2.1 \cdot 10^{-5} \mathrm{~m} \mathrm{~Pa}^{-1}, \beta=0.67, \tau_{2}=6.38 \cdot 10^{2} \mathrm{~d}, \alpha=24 \mathrm{Kg} \mathrm{m}^{-4} \mathrm{~s}^{-1}$. Timescales for well B: $t_{f}=10.8 \mathrm{~d}, t_{m}=54.1 \mathrm{~d}$. Model parameters for well C (red line): fixed parameters: $r_{w}=0.09 \mathrm{~m}$, $N_{f}=48$; fitted parameters: $R=34.6 \mathrm{~m}, S_{f}=3.6 \cdot 10^{-6} \mathrm{~m} \mathrm{~Pa}^{-} 1, T_{f}=1.4 \cdot 10^{-9} \mathrm{~m}^{3} \mathrm{~Pa}^{-1} \mathrm{~s}^{-1}$, $\Delta p=3.6 \cdot 10^{7} \mathrm{~Pa}, S_{m}=3.1 \cdot 10^{-5} \mathrm{~m} \mathrm{~Pa}^{-} 1, \beta=0.5, \tau_{2}=4.9 \cdot 10^{4} \mathrm{~d}, \alpha=2.5 \mathrm{Kg} \mathrm{m}^{-4} \mathrm{~s}^{-1}$. Timescales for well C: $t_{f}=35.6 \mathrm{~d}, t_{m}=342.4 \mathrm{~d}$.

\subsection{Parameter Sensitivity}

Here we perform a sensitivity analysis to evaluate the control level of each parameter, or group of parameters, in the multi-continuum model. The sensitivity analysis consists in changing on parameter while keeping the others fixed. We perform this analysis starting from the parameter set best-fitted for well A (see Fig.3). Figure 5 displays the variation of the gas flowrate dynamic versus the radius of 


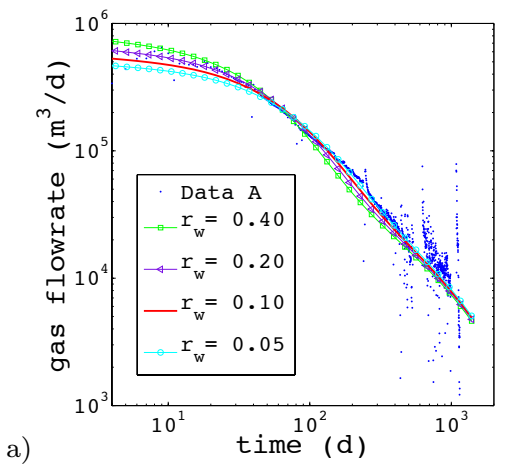

b)

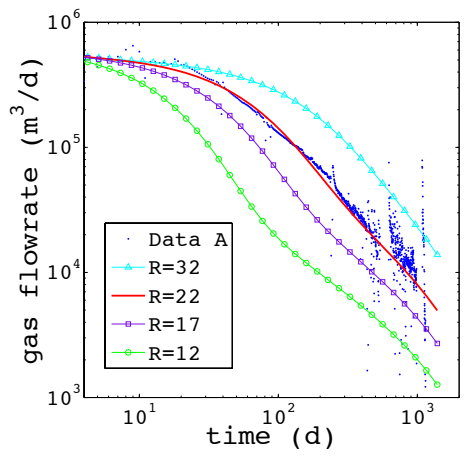

Fig. 5 Previous well A flowrate fit (Fig.3) for different values of the radius of the horizontal well $r_{w}$ (on the left, Fig. 5a) and of the extension of the fractured zone $R$ (on the right, Fig. $5 \mathrm{~b})$. The values of $r_{w}$ and $R$ are indicated in the figures and expressed in meters. Timescales: $t_{f}=6.5 \mathrm{~d}$ for $R=12 \mathrm{~m}, t_{f}=13 \mathrm{~d}$ for $R=17 \mathrm{~m}, t_{f}=22 \mathrm{~d}$ for $R=22 \mathrm{~m}$ and $t_{f}=46 \mathrm{~d}$ for $R=32 \mathrm{~m}$. Values of the others parameters are given in the caption of Fig.3.

a)


Fig. 6 Previous well A flowrate fit (Fig.3) for different values of the storativity of the fractured zone $S_{f}$ (on the left, Fig. 6a) and the transmissivity of the fractured zone $T_{f}$ (on the right, Fig. $6 \mathrm{~b}$ ). The values of $T_{f}$ indicated in the figure are expressed in $\mathrm{m}^{2} / \mathrm{d}$. Others parameters value are given in the caption of Fig.3.

the horizontal well $r_{w}$ (Fig.5a) and the radial extension of the fractured zone $R$ (Fig.5b).

Figure 5a, shows that the initial value of the gas flowrate slightly increases with the radius of the horizontal well, but the shape of the curves or in other words the dynamic of the gas flowrate and the late time behavior are almost unaltered. Conversely, Figure 5b shows that the initial value of the gas flowrate does not change for different amplitudes of the fractured zone. The curves plotted for different values of $R$ from $12 \mathrm{~m}$ to $32 \mathrm{~m}$ overlap at early times but separate later. Different values for $R$ induce different timescale $t_{f}$, i.e. mean depletion times of the fractured zone.

As pointed out previously, the gas flux starts decaying for $t>t_{f}$. Figure 6 displays the variation of the flowrate as a function of the total compressibility of the fractured zone $S_{f}$ (Fig.6a) and the effective transmissivity of the fractured 

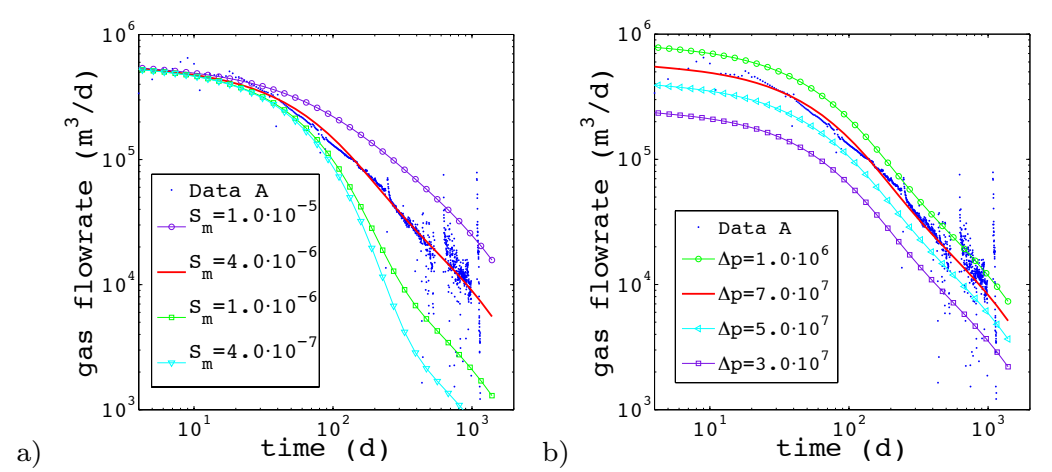

Fig. 7 Previous well A flowrate fit (Fig.3) for different values of the storativity of the matrix $S_{m}$ (Fig.3a) and different values of the difference between the initial pressure and the pressure in the well $\Delta p=\bar{p}_{f}(0)-p_{w}$ (Fig.3a). The values of $S_{m}$ and $\Delta p$ are indicated in the figures. Others parameters value are given in the caption of Fig.3.

zone $T_{f}$ (Fig.6b). As observed when varying $R$ (Fig.5b), varying $S_{f}$ and $T_{f}$ implies different $t_{f}$, and thus depletion scales.

Thus, higher values of total compressibility of the fractured zone imply more persistent high flowrates. In fact, higher $S_{f}$ implies higher storativity and larger amount of gas trapped. For large times, the curves tend to the same behaviour. This is because at large times the behaviour is controlled by the parameters of the matrix. As for $R$, at very short times, $S_{f}$ does not seem to have a strong influence of the value of the gas flowrate. Conversely Figure $6 \mathrm{~b}$, shows that the initial value of the gas flowrate is very dependent on $T_{f}$ and varies proportionally. As for $S_{f}$, and for the same reason, at larger times, the curves for different $T_{f}$ get closer and share similar behaviour. Figure 7a shows the dependency of the flowrate curves on the total compressibility of the matrix $S_{m}$. As expected, $S_{m}$ influences the long time behaviour and does not influence at all short time behaviour (the opposite to the parameters of the fractured zone). For $t>\tau_{f}$ higher $S_{m}$ implies higher long time flowrate, because the amount of gas to extract is higher. For extremely low compressibility in the matrix, once the fractured zone is depleted, the flowrate decreases exponentially, similarly to the homogeneous model illustrated in Fig. 3. Figure 8 displays flowrate curves for different parameters of the power law distribution of transition times in the matrix given in Eq. (19): 8a for different exponents $\beta, 8 \mathrm{~b}$, for different cutoff $\tau_{2}$. Since the $\beta$ and $\tau_{2}$ parameters characterize the matrix, neither of them influences the initial dynamic of the gas flowrate, but only the long time behaviour. The parameter $\beta$ weakly influences the slope of the gas flowrate for that decline regime. For higher values of $\beta$ we can observe a slightly sharper decrease. The reason is that decreasing $\beta$ implies flatter distribution of mass transfer times, while a flatter distribution of mass transfer times triggers a longer tail in the declining flowrate. The influence of $\beta$ is clearly visible on the figure when $\tau_{2} \gg t_{f}$, i.e. the asymptotic regime $t^{-\beta}$ is reached, as shown in Figure2 and discussed above.

The parameter $\tau_{2}$ controls significantly the behaviour of the system at long times. Figure $8 \mathrm{~b}$ displays different shapes of the flowrate decline curves. Small $\tau_{2}$ implies that the distribution of transition times in the matrix is narrow. A 
)
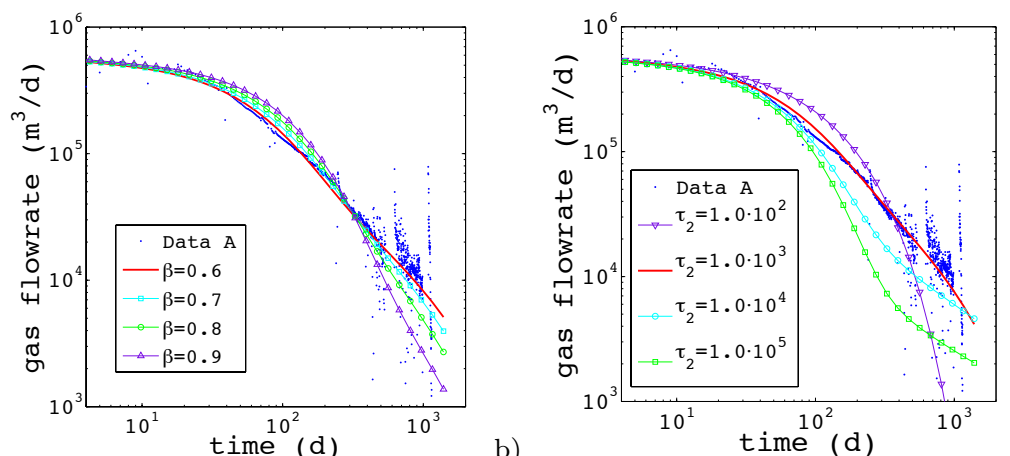

Fig. 8 Previous well A flowrate fit (Fig.3) for different values of the exponent $\beta$ (on the left, Fig.8a) and of cut-off time $\tau_{2}$ (on the right, Fig.8b) of the power law distribution given in (19). The values of $\tau_{2}$ indicated in the figure are expressed in days. Others parameters value are given in the caption of Fig. 3 .

distribution of transition times can be mapped into a distribution of transmissivities. Small transition times imply high transmissivities. Thus, a small value of $\tau_{2}$ implies a narrow distribution of transmissivities characterized by relatively high values. For high values of transmissivities the matrix reaches equilibrium relatively fast and the whole system behaves as an equivalent homogeneous system and we do not observe the long time scaling of the flowrate. This is the case of the curve of Fig. $8 \mathrm{~b}$ with $\tau_{2}=10^{2} \mathrm{~d}$, that behaves similarly to the curve of the equivalent homogeneous model with $\varphi=S_{m}$ in Fig. 3 right. For larger $\tau_{2}$ (see curve with $\tau_{2}=10^{3} \mathrm{~d}$ ), the distribution of storage times is broad enough and non-equilibrium mass transfer between the fractured zone and the matrix induces anomalous scaling. As $\tau_{2}$ becomes even larger (see curve with $\tau_{2}=10^{4} \mathrm{~d}$ and $\tau_{2}=10^{5} \mathrm{~d}$ ) a third behaviour occurs. Very high cut offs imply extremely low permeability of the matrix. Thus, for a long time, the contribution of the matrix does not affect the total gas flowrate, and the system behaves as the homogeneous model with $\varphi=0$ (see Fig. 3). Yet, contrary to the equivalent homogeneous model (Fig. 3), the matrix contributes to the dynamics of the system at large times. The parameter $\tau_{2}$ is a key parameters because the decline curve decreases exponentially, and thus production drop noticeably, for time larger than $\tau_{2}$.

In Fig.7b we explore the difference between the pressure in the well and the initial pressure. We observe that the initial pressure difference does not affect the dynamics of the gas flowrate but only shift it upward (for higher difference of pressure) or downward (for lower difference). Accordingly, it appears that the initial pressure of shale matrix can be determined as soon as the shape is fitted by set of parameter describing the reservoir hydrodynamic properties. We found an initial pressure of $10.40 \cdot 10^{7} \mathrm{~Pa}, 7.28 \cdot 10^{7} \mathrm{~Pa}$ and $4.48 \cdot 10^{7} \mathrm{~Pa}$ for well $\mathrm{A}, \mathrm{B}$ and $\mathrm{C}$ respectively that corresponds to $1.10,0.77$ and 0.85 times the lithostatic pressure respectively.

It turns out that the model derived is slightly sensitive to the parameter $\alpha$, the skin factor, but only at very short time. Evaluating the terms in the equation for the flux Eq.(32), we observe that the term A, which contains the parameter $\alpha$ multiplies the difference of the product of two Bessel functions which actually 
gives very small values compared to the first term in the denominator. Physically speaking this means that only at very early times, the flux is limited by the skin factor, at larger times by the release flux from the matrix.

\section{Conclusion}

In this work we have derived an original analytical multi-continuum model that can be used for analyzing gas flowrates in shale reservoirs. We show that both the low permeability and the intrinsic heterogeneity of the shale matrix control the complex transient regimes and the long asymptotic decreasing flowrate usually observed during the gas production. The model describes the dynamics of the gas flowrate as the function of the standard physical parameters (compressibility, transmissivity, ...) and the (simplified) geometry of the stimulated reservoir. Because of the manifest lack of information on the (mechanically altered) shale matrix and consequently the spatial distribution of its heterogeneity, we described the matrix diffusivity stochastically using a distribution of transition times. The latter is parametrized by $\tau_{2}$ and $\beta$, the cut off and the slope of the distribution, respectively.

The model predicts:

1) an initial regime controlled by the properties of the transmissivity $T_{f}$ and diffusivity $T_{f} / S_{f}$ of the fractures, as well as the length of the individual fracture $R$,

2) a second transient regime, which covers the main production duration of the well, where the flowrate is controlled by coupled effects of both the fractures and the shale matrix properties; this regime corresponds to the refill of the fractures by the matrix. This part of the gas production curve can display distinctly different trends including power law decline regimes: $Q(t) \propto\left(t / \tau_{2}\right)^{-\beta}$ where $\beta \in(1 / 2 ; 1)$ is a lumped parameter describing the heterogeneity distribution in the matrix.

3) For $t \gg \tau_{2}$ the gas production rate decreases exponentially: $Q(t) \propto \exp \left(-t / \tau_{c}\right)$ with $\tau_{c} \sim R^{2}\left(S_{f}+S_{m}\right) / T_{f}$.

It is worth noticing that for $\beta=0.5$ the presented model shows the scaling $Q(t) \propto t^{-1 / 2}$ for the gas flux, which is typical for a dual continuum model characterized by a homogeneous matrix domain such as the one considered by [8]. These authors account for non-linear pressure diffusion within the matrix domain, which however does not alter the characteristic $t^{-1 / 2}$-behavior. The model proposed in this paper accounts for a heterogeneous matrix domain and thus allows for different scaling behaviors.

To illustrate the applicability of the model we considered real data from three production wells that display distinctly different gas flowrate behaviours. We show that the model can accurately fit the different flowrate behaviours and that the parameter values obtained by the fitting procedure are in accordance with typical ones found in the shale reservoir literature.

As for all multi-parameter models, the reliability of the results increases if one can constrain some parameters and, or, impose a restricted range of variability. In our model one have six parameters which are often poorly known $R, S_{f}, T_{f}, S_{m}, \beta, \tau_{2}$. Any reliable data concerning these parameters will dramatically reduce the uncertainties on the parameter estimation and consequently on the predictability of this tool. For instance, a good estimation of $R$ could be surely 
obtained by micro-seismic. Likewise, a better knowledge of the fracture properties from geophysical or experimental data will improve the best fitting of the short time behavior, while (multi-scale) measurements of the matrix dispersivity would be of great importance for long term predictions. Yet, the reliability of such measurements on mechanically extracted cores may be arguable and an accurate evaluation of the actual effect of fracking on the matrix properties remains highly challenging. Accordingly, we believe that our model may be useful for tackling the matrix properties once the other parameters, including the fracture properties, are independently estimated.

\section{A Derivation of the memory function}

We assume that the lower cut-off scale $\tau_{1}$ in (19) is much smaller than the upper cut-off $\tau_{2}$ so that $\tau_{1} / \tau_{2} \ll 1$ and we approximate

$$
P_{i m}(\tau)=\frac{1-\beta}{\tau_{2}}\left(\frac{\tau}{\tau_{2}}\right)^{-\beta} \Theta\left(\tau_{2}-\tau\right)
$$

Inserting (41) in (18), the memory function for a multi-continuum model with a constant retardation factor reads:

$$
\varphi(t)=S_{i m} \frac{1-\beta}{\tau_{2}}\left(\frac{t}{\tau_{2}}\right)^{-\beta} \int_{t / \tau_{2}}^{\infty} d z z^{\beta-1} g^{\prime}(z),
$$

where $g^{\prime}(z)$ is defined as: $g(t, \tau)=\frac{1}{\tau} g^{\prime}\left(\frac{t}{\tau}\right)$. The local memory function $g(t, \tau)$ can be expressed as above from the definition of $\tilde{g}(\omega, \tau)$ given in $(11)$, we note that $\tilde{g}(\omega, \tau)$ is precisely a function of the product $\omega \tau$. The function $g^{\prime}(z)$ behaves as $z^{-1 / 2}$ for $z<1$ and decays exponentially fast for $z \gg 1$. Thus, we approximate it here by the truncated power-law distribution: $g^{\prime}(z)=z^{-1 / 2} \exp (-z) / \Gamma(1 / 2)$. Inserting the previous approximation into (42) and executing the integral for the memory function $\varphi_{\kappa}(t)$ reads

$$
\varphi(t)=S_{i m} \frac{1-\beta}{\tau_{2} \Gamma(1 / 2)}\left(\frac{t}{\tau_{2}}\right)^{-\beta} \Gamma\left(\beta-1 / 2, t / \tau_{2}\right) .
$$

This function behaves as a power-law according to $t^{-\beta}$ for $t \ll \tau_{2}$ and is cut-off exponentially fast for $t \gg \tau_{2}$. Thus, we approximate it by the truncated power-law given in (20).

\section{B General solution of the model}

The general solution of Eq. (15) is given by a combination of modified Bessel functions of first kind $\mathcal{I}_{0}(\cdot)$ and second kind $\mathcal{K}_{0}(\cdot)[36]$ :

$$
\left\langle\bar{p}_{f}(r, \lambda)\right\rangle=N(\lambda) \mathcal{K}_{0}\left[r \sqrt{\lambda \tau^{\prime}(\lambda)}\right]+M(\lambda) \mathcal{I}_{0}\left[r \sqrt{\lambda \tau^{\prime}(\lambda)}\right]+\frac{\bar{p}_{f}(r, 0)}{\lambda},
$$

with $\tau^{\prime}(\lambda)$ defined in Eq. (25). The functions $N(\lambda)$ and $M(\lambda)$ do not depend on the radial coordinate $r$ and are determined by imposing the given boundary condition in the general solution (44). Considering no radial flux at $r=R$, Eq. (27), we obtain: $N(\lambda)=M(\lambda) \mathcal{I}_{1}\left[r \sqrt{\lambda \tau^{\prime}}\right] / \mathcal{K}_{1}\left[r \sqrt{\lambda \tau^{\prime}}\right]$ and by imposing Cauchy-type boundary condition at the well, Eq. (26), we obtain $M(\lambda)$. By inserting $N(\lambda)$ and $M(\lambda)$ in Eq.(44) and rearranging the terms we obtain Eq.(23). 


\section{Asymptotic expansion}

In order to evaluate the asymptotic behavior of the gas flux $Q(t)$, we consider the expansion of the Bessel functions in (32) for small arguments $x$,

$$
\begin{aligned}
\mathcal{K}_{0}(x) & =-\ln (x / 2)+\ldots \\
\mathcal{I}_{0}(x) & =1+\ldots \\
\mathcal{K}_{1}(x) & =\frac{1}{x}+\frac{1}{2} \ln x \ldots \\
\mathcal{I}_{1}(x) & =\frac{x}{2} \ldots,
\end{aligned}
$$

where the dots denote sub-leading contributions. Substituting these expansion into (32) we obtain:

$$
Q^{*}(\lambda) \simeq \frac{\pi T_{f}\left({R^{\prime}}^{2}-{r^{\prime}}_{w}^{2}\right)\left[\frac{p_{f}(0)}{\lambda}-p_{w}^{*}(\lambda)\right]}{1+\frac{R^{\prime 2}}{2} \ln \frac{R^{\prime}}{r_{w}^{\prime}}+\frac{A}{2}\left({R^{\prime}}^{2}-r_{w}^{\prime 2}\right)}
$$

Setting the pressure in the extraction well constant, i.e., $p_{w}(t)=p_{w}=$ constant, which implies $p_{w}^{*}(\lambda)=\lambda^{-1} p_{w}$, and substituting $r^{\prime}, R^{\prime}$ and $r_{w}^{\prime}$ by (24) and (29) we obtain Eq.(33).

\section{Nomenclature}

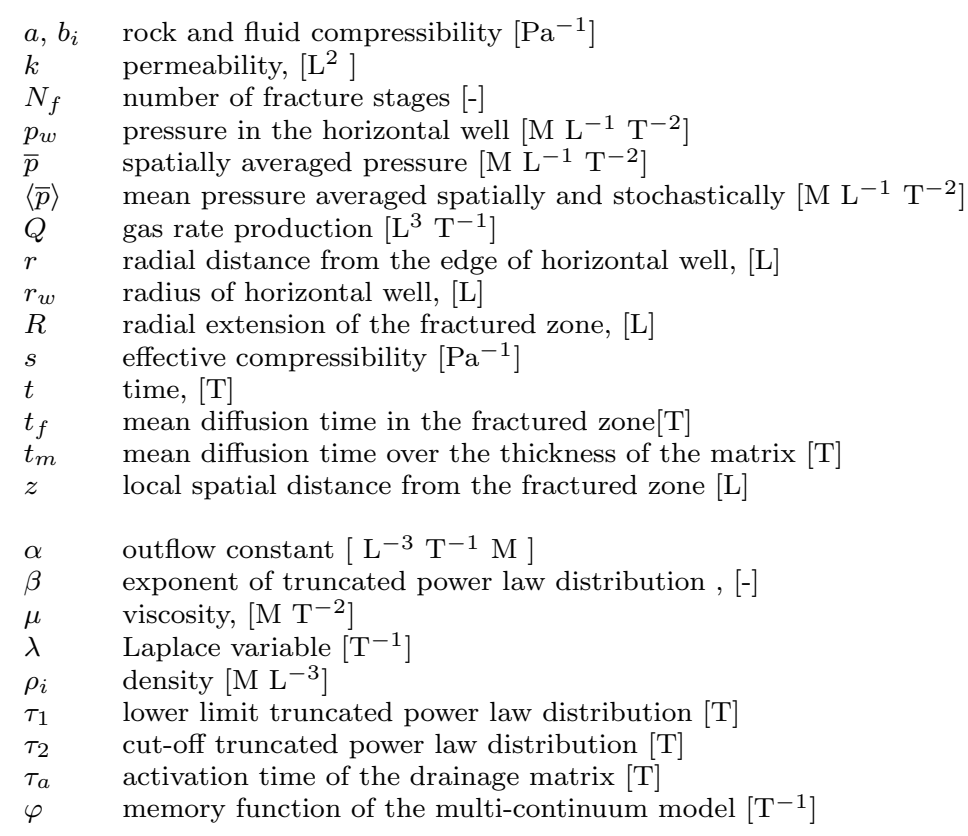

Derived quantities:

$K_{i}=k_{i} / \mu_{i}$

$S_{i}=z_{i} s_{i}$

permeability divided by viscosity, $\left[\mathrm{L}^{3} \mathrm{M}^{-1} \mathrm{~T}\right]$

$T_{i}=z_{i} K_{i}$

total effective compressibility, $\left[\mathrm{m} \mathrm{Pa}^{-1}\right]$

effective transmissivity $\left[\mathrm{L}^{4} \mathrm{M}^{-1} \mathrm{~T}\right]$ 


\section{E Acknowledges}

The authors gratefully acknowledge the financial support for this project from Imperial College, London, Joint Industry Project on Well Test Analysis in Complex Fluid-Well-Reservoir Systems, funded by BG Group, BHP-Billiton, Petro SA, Schlumberger and Eon-Rurhgas. They are grateful to BG Group and BHP-Billinton for providing the data used in this study. M. D. acknowledges the support of the European Research Council (ERC) through the project MHetScale (contract number 617511).

\section{References}

1. J. Arps, SPE-945228-G Trans. AIM(160), 228 (1945)

2. W.J. Lee, R.E. Slide, in SPE-130102, vol. 130102 (2010), vol. 130102

3. D. Ilk, A. Perego, J. Rushing, T. Blasingame, in SPE-116731, vol. 116731 (2008), vol. 116731

4. A. Araya, E. Ozkan, SPE-77690 77690 (2002)

5. L. Mattar, B. Gault, K. Morad, C.R. Clarkson, C.M. Freeman, SPE 119897 (2008)

6. F. Medeiros, B. Kurtoglu, M. Oil, E. Ozkan, SPE Reservoir Evaluation \& Engineering 110848(June), 559 (2010)

7. M.J. Fetkovich, SPE, Journal of Petroleum Technology pp. 1065-1077 (1980)

8. T.W. Patzek, F. Male, M. Marder, Proceedings of the National Academy of Sciences of the United States of America 110(49), 19731 (2013). DOI 10.1073/pnas.1313380110. URL http://www.pubmedcentral.nih.gov/articlerender.fcgi?artid=3856843\&tool=pmcentrez\&rendertype $=$ abstract

9. S. Maxwell, C. Waltman, N. Warpinski, N. Mayerhofer, M.J., Boroumand, in SPE-102801, vol. 102801 (2006), vol. 102801

10. G.I. Barenblatt, I.P. Zheltov, I.N. Kochina, PMM 24(5), 825 (1960)

11. J.E. Warren, P.J. Root, The Soc. Petrol. Eng. J. 3(3), 245 (1963)

12. D. Bourdet, A.C. Gringarten, in SPE Annual Technical Conference and Exhibition, 21-24 September, Dallas, Texas (Society of Petroleum Engineers, Dallas, Texas, 1980). DOI 10.2118/9293-MS

13. C.R. Dykhuizen, Water Resources 21(12), 1531 (1987)

14. R.R. Peters, E.A. Klavetter, Water Resources Research 24(3), 416 (1988)

15. M. Bai, D. Elsworth, J.C. Roegiers, Water Resources Research 29(6), 1621 (1993)

16. M.J. Gatens, W.J. Lee, H.S. Lane, A.T. Watson, D.K. Stanley, Journal of Petroleum Technology 41(5), 519 (1989)

17. A.T. Watson, M.J. Gatens, J.M. Lee, Z. Rahim, SPE Reservoir Engineering 5(3) (1990)

18. K. Serra, a.C. Reynolds, R. Raghavan, a.C. Reynolds, Journal of Petroleum Technology 35(12) (1983). DOI 10.2118/10780-PA. URL http://www.onepetro.org/mslib/servlet/onepetropreview?id=00010780\&soc $=\mathrm{SPE}$

19. E. Carlson, J. Mercer, Journal of Petroleum Technology 43(4) (1991). DOI 10.2118/19311-PA. URL http://www.onepetro.org/mslib/servlet/onepetropreview?id=00019311\&soc=SPE

20. K.L. Kuhlman, B. Malama, J.E. Heath, Water Resour. Res. 51(2), 848 (2015). DOI DOI: 10.1002/2014WR016502

21. A.H. El-Banbi, Analysis of Tight Gas Well Performance. Ph.D. thesis (1998)

22. R.O. Bello, R.A. Wattenbarger, SPE, Journal of Canadian Petroleum Technology 49(12), 143229 (2010). DOI 10.2118/143229-PAContent

23. I. Neuweiler, D. Erdal, M. Dentz, Vadose Zone Journal 11, 0 (2012). DOI $10.2136 / \mathrm{vzj} 2011.0132$

24. J. Tecklenburg, I. Neuweiler, M. Dentz, J. Carrera, S. Geiger, C. Abramowski, O. Silva, Advances in Water Resources 62, 475 (2013). DOI 10.1016/j.advwatres.2013.05.012. URL http://dx.doi.org/10.1016/j.advwatres.2013.05.012

25. S. Geiger, M. Dentz, I. Neuweiler, SPE Journal 18(8), 670 (2013). DOI 10.2118/148130MS. URL https://www.onepetro.org/journal-paper/SPE-148130-PA

26. J. Bear, Dynamics of fluids in porosu media, dover edn. (American Elsevier Publishing Company, New York, 1972)

27. R. Haggerty, S.M. Gorelick, Water Resources Research 31(10), 2383 (1995)

28. J. Carrera, X. Sánchez-Vila, I. Benet, A. Medina, G. Galarza, J. Guimerà, Hydrogeology Journal 6(1), 178 (1998). DOI $10.1007 / \mathrm{s} 100400050143 . \quad$ URL http://www.springerlink.com/openurl.asp?genre=article\&id=doi:10.1007/s100400050143 
29. M. Abramowitz, I. Stegun, Handbook of Mathematical Functions with Formulas, Graphs, and Mathematical Tables (Dover Publications, New York, 1965)

30. K.J. Hollenbeck. INVLAP.M: A matlab function for numerical inversion of Laplace transforms by the Hoog algorithm (1998). URL http://www.isva.dtu.dk/staff/karl/invlap.htm

31. A. Cortis, B. Berkowitz, Ground Water 43(6), 947 (2005). DOI 10.1111/j.17456584.2005.00045.x

32. B. Berkowitz, A. Cortis, M. Dentz, H. Scher, Rev. Geophys. 44(RG2003), RG2003 (2006). DOI 10.1029/2005RG000178.1.INTRODUCTION

33. D.G. Friend, J.F. Ely, H. Ingham, J.Phys. Chem. Ref. Data 18(2), 583 (1989)

34. C.E. Neuzil, Water Resources Research 30(2), 145 (1994). DOI 10.1029/93WR02930. URL http://doi.wiley.com/10.1029/93WR02930

35. W. Yu, K. Sepehrnoori, Proceedings of 2013 SPE Production and Operations Symposium 2013 (2013). DOI 10.2118/164509-MS. URL https://www.onepetro.org/conferencepaper/SPE-164509-MS

36. H.S. Carslaw, J.C. Jaeger, Conduction of Heat in Solids, vol. 36 (Oxford University Press, Oxford, 1947). DOI 10.2307/3610347 